\title{
SYNTHESIS AND ANTIBACTERIAL ACTIVITY OF 3-(2-(5- AMINO-1H-PYRAZOL-4-YL) THIAZOL-4-YL)-2H-CHROMEN- 2-ONE AND ITS DERIVATIVES
}

\author{
M. Shivanand ${ }^{1,2}$, P.Vijaya Kumar ${ }^{3}$ and V. Ravikumar ${ }^{1 *}$ \\ ${ }^{1}$ Department of Chemistry, Kamala Institute of Technology and Science, Huzurabad, \\ Karimnagar-505468(Telangana), India \\ ${ }^{2}$ Department of Chemistry,Rayalaseema University,Kurnool-518 007(Andhra Pradesh), India. \\ ${ }^{3}$ Department of Chemistry, National Institute of Technology, \\ Warangal-506 004(Telangana), India. \\ *E-Mail: vemularavi97@gmail.com
}

\begin{abstract}
A simple and convenient method for the preparation of 3-(2-(5-amino-1H-pyrazole-4-yl)thiazol-4-yl)-2H-chromene2-one and 3-(2-(5-amino-3-methyl-1H-pyrazole-4-yl)thiazol-4-yl)-2H-chromene-2-ones (3a-f and 5a-f) have been prepared in good yields. The compounds have been synthesized from 2-(4-(2-oxo-2H-chromene-3-yl)thiazol-2yl)acetonitrile (1a-f), with DMF-DMA, DMA-DMA and hydrazine hydrochloride in ethanol resulted in the formation of title compounds. In this regard the whole compounds were tested for promising antibacterial activity, characterized by analytical and spectral data.
\end{abstract}

Keywords: Coumarin, Thiazole, Pyrazole, Thiazolyl-pyrazoles, Cyclisation reaction.

@ RASĀYAN. All rights reserved

\section{INTRODUCTION}

Thiazolyl-pyrazole ${ }^{1}$ derivatives have been shown remarkable therapeutic applications in medicinal chemistry and pharmaceutical chemistry. Previously, thiazoles were synthesized in the year 1887 by Hantzsch and Weber.These are a significant set of heterocyclics, to establish in a lot of active drugs such as Sulfathiazole (antimicrobial), Abafungin (antifungal), Tiazofurin (antineoplastic) and Ritonavir (antiretroviral). Hence, it is present in many compounds, it shows important biological activities such as antihypertensive $^{1}$, antimicrobial, antifungal ${ }^{2}$, anticancer ${ }^{3}$, anti-inflammatory ${ }^{4}$, anti-HIV ${ }^{5}$, antidiabetic ${ }^{6}$, and antconvulsant ${ }^{7}$ activities. Pyrazoles are an essential class of heterocyclics in medicinal chemistry, which represent the basic frame-work of drugs such as Celecoxib and as well studied heterocyclic compounds with huge therapeutic value ${ }^{8}$. Consequently, many pyrazoles have been displayed a variety of biological activities $^{9-13}$ such as antitubercular, antiviral, antitumor and anticancer properties. All these derivatives have been considered as adaptable building blocks and intermediates. The earlier study reports as well derived from coumarin. ${ }^{14-15}$ During this protocol we have developed the latest heterocyclic systems on $3^{\text {rd }}$ position of coumarin.

Coumarin derivatives are widely distributed in several drugs, and the literature study reports of entire coumarin derivatives shown important biological activities among them are anti-microbial, ${ }^{16-17}$ antifungal, ${ }^{18}$ anti-inflammatory, ${ }^{19}$ anticancer, ${ }^{20-21}$ anti-tubercular, ${ }^{22}$ and antitumor activity. ${ }^{23}$ The earlier literature work reveals that the synthesis of different kind of heterocyclic systems derived from coumarin. ${ }^{14-15}$ It appears to be valuable to synthesize a few coumarin containing derivatives of 3-(2-(5amino-1H-pyrazole-4-yl)thiazol-4-yl)-2H-chromene-2-one and 3-(2-(5-amino-3-methyl-1H-pyrazole-4yl)thiazol-4-yl)-2H-chromene-2-one. In view that herein we report the synthesis and antibacterial activity of title compounds and its derivatives.

Rasayan J. Chem., 12(4), 1810-1815(2019)

http://dx.doi.org/10.31788/RJC.2019.1245375

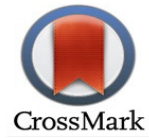




\section{RASĀYAN J. Chem.}

GALLEY PROOFS

\section{Materials and Methods}

\section{EXPERIMENTAL}

All the chemicals were purchased from viable sources, used without purification. The prepared materials were checked by TLC on silica plates (E-Merk, Mumbai, India). Melting points were checked with an open capillary tube with a "Centex" melting point apparatus, Mumbai, India and were uncorrected. IR spectra were recorded in $\mathrm{KBr}$ pellets on a Bruker WM-200 MHz spectrometer. ${ }^{1} \mathrm{H}$-and ${ }^{13} \mathrm{C}$-NMR spectra were documented on a Bruker WM-400 spectrometer (in $\delta \mathrm{ppm}$ ) using TMS as an internal standard. The mass spectra (EI-MS) were determined by Jeol-D-300 spectrometer at $70 \mathrm{eV}$. CHN analyses were recorded on Carlo Erba 1108 Heraeus analyzer. The 3-(2-bromoacetyl)chromen-2-ones were prepared by reported procedures ${ }^{15}$ which on further reaction with thioacetamide in ethanol to gave 2-(4-(2-oxo- $2 \mathrm{H}$ chromene-3-yl)thiazol-2-yl)acetonitrile (1a-f).

Synthesis of (E)-3-(dimethylamino)-2-(4-(2-oxo-2H-chromene-3-yl)thiazol-2-yl)acrylonitrile(2a-f) A mixture of 2-(4-(2-oxo-2H-chromene-3-yl)thiazol-2-yl)acetonitrile 1a-f (1 mmol), N,N-Dimethyl formamide dimethyl acetal (DMF-DMA) $(1.5 \mathrm{mmol})$ in toluene was refluxed for 2 hours. Then the reaction mixture was checked by TLC, after completion of reaction the solvent was distilled out and added hexane to get the crude solid, was filtered, dried and recrystallized from Hexane/EtOAc.

\section{(E)-3-(dimethylamino)-2-(4-(2-oxo-2H-chromen-3-yl)thiazol-2-yl)acrylonitrile 2a}

Brown solid.Yield 90\%. m.p. $253-255^{\circ} \mathrm{C}$. IR (KBr): $1607(-\mathrm{C}=\mathrm{N}), 1720$ (lactone, $\left.-\mathrm{C}=\mathrm{O}\right) \quad \mathrm{cm}^{-1} ;{ }^{1} \mathrm{H}$ NMR (400 MHz, DMSO- $\left.d_{6}\right): \delta 2.84\left(\mathrm{~s}, 6 \mathrm{H}, 2-\mathrm{CH}_{3}\right), 6.86(\mathrm{~s}, 1 \mathrm{H},-\mathrm{C}=\mathrm{CH}), 7.32-7.48\left(\mathrm{~m}, 2 \mathrm{H}, \mathrm{Ar}-\mathrm{H}\right.$ of $\mathrm{C}_{6} \&$ $\left.\mathrm{C}_{8}-\mathrm{H}\right), 7.64\left(\mathrm{~m}, 1 \mathrm{H}, \mathrm{Ar}-\mathrm{H}\right.$ of $\left.\mathrm{C}_{7}-\mathrm{H}\right), 7.78\left(\mathrm{~d}, 1 \mathrm{H}, \mathrm{C}_{5}-\mathrm{H}\right.$ of $\left.\mathrm{Ar}-\mathrm{H}, J=9 \mathrm{~Hz}\right), 8.16\left(\mathrm{~s}, 1 \mathrm{H}, \mathrm{C}_{5}{ }^{\prime}-\mathrm{H}\right.$ of thiazole $)$, $8.68\left(\mathrm{~s}, 1 \mathrm{H}, \mathrm{C}_{4}-\mathrm{H}\right.$ of coumarin); ${ }^{13} \mathrm{C}$ NMR $\left(400 \mathrm{MHz}, \mathrm{DMSO}-d_{6}\right): \delta 45.4,86.8,116.5,118.6,121.4$, $124.2,125.8,127.8,128.5,129.5,149.2,156.7,158.9,164.6,166.5, \mathrm{MS}: \mathrm{m} / z$ 323. Anal. Calcd for $\mathrm{C}_{17} \mathrm{H}_{13} \mathrm{~N}_{3} \mathrm{O}_{2} \mathrm{~S}: \mathrm{C}, 63.14 ; \mathrm{H}, 4.05 ; \mathrm{N}, 12.99$. Found: $\mathrm{C}, 63.10 ; \mathrm{H}, 4.02 ; \mathrm{N}, 12.96 \%$.

\section{Synthesis of 3-(2-(5-amino-1H-pyrazole-4-yl)thiazol-4-yl)-2H-chromene-2-one(3a-f)}

To a solution of (E)-3-(dimethylamino)-2-(4-(2-oxo-2H-chromene-3-yl)thiazol-2-yl)acrylonitrile 2a-f (1 $\mathrm{mmol})$, in ethanol was added hydrazine hydrochloride $(1 \mathrm{mmol})$ was refluxed for 8 hours. After that checked the reaction mixture by TLC, then solvent was distilled out under reduced pressure to get the residue to this added methyl tertiary butyl ether and filtered insoluble material. This organic layer was washed with $10 \% \mathrm{NaOH}$ solution, followed by dil. $\mathrm{HCl}$ and water. This was dried in sodium sulfate and concentrated on getting the crude compound was recrystallized from toluene/EtOAc.

\section{Spectral Characterization}

3-(2-(5-Amino-1H-pyrazol-4-yl)thiazol-4-yl)-2H-chromen-2-one 3a

White solid. Yield 86\%. m.p. $260-262^{\circ} \mathrm{C}$. IR (KBr): $1607(-\mathrm{C}=\mathrm{N}), 1719$ (lactone, $\left.-\mathrm{C}=\mathrm{O}\right)$ and 3418 (pyrazole, $-\mathrm{NH}) \mathrm{cm}^{-1} ;{ }^{1} \mathrm{H}$ NMR $\left(400 \mathrm{MHz}, \mathrm{DMSO}-d_{6}\right)$ : $\delta 5.41\left(\mathrm{br}, 2 \mathrm{H},-\mathrm{NH}_{2}\right), 7.34-7.46(\mathrm{~m}, 2 \mathrm{H}, \mathrm{Ar}-\mathrm{H}$ of $\left.\mathrm{C}_{7} \& \mathrm{C}_{8}-\mathrm{H}\right), 7.61\left(\mathrm{~m}, 1 \mathrm{H}, \mathrm{Ar}-\mathrm{H}\right.$ of $\left.\mathrm{C}_{6}-\mathrm{H}\right), 7.74\left(\mathrm{~d}, 1 \mathrm{H}, \mathrm{C}_{5}-\mathrm{H}\right.$ of $\left.\mathrm{Ar}-\mathrm{H}, J=9 \mathrm{~Hz}\right), 7.79(\mathrm{~s}, 1 \mathrm{H}, \mathrm{NH}$ Pyrazole), $8.11\left(\mathrm{~s}, 1 \mathrm{H}, \mathrm{C}_{5}{ }^{\prime}-\mathrm{H}\right.$ of thiazole), $8.68\left(\mathrm{~s}, 1 \mathrm{H}, \mathrm{C}_{4}-\mathrm{H}\right.$ of coumarin); ${ }^{13} \mathrm{C}$ NMR $\left(400 \mathrm{MHz}, \mathrm{DMSO}-d_{6}\right): \delta 91.3$, 116.9, 121.4, 124.1, 125.8, 127.8, 128.5, 129.5, 132.9, 134.8, 146.0, 149.6, 153.9, 156.7, 166.5, MS: $\mathrm{m} / \mathrm{z}$ 310. Anal. Calcd for $\mathrm{C}_{15} \mathrm{H}_{10} \mathrm{~N}_{4} \mathrm{O}_{2} \mathrm{~S}: \mathrm{C}, 58.05 ; \mathrm{H}, 3.25 ; \mathrm{N}, 18.05$. Found: C, 58.01; H, 3.21; N, 18.01\%.

\section{3-(2-(5-amino-1H-pyrazol-4-yl)thiazol-4-yl)-8-methoxy-2H-chromen-2-one 3b}

White solid. Yield 84\%. m.p. $255-257^{\circ} \mathrm{C}$. IR (KBr): $1608(-\mathrm{C}=\mathrm{N}), 1721$ (lactone, $\left.-\mathrm{C}=\mathrm{O}\right)$ and 3416 (pyrazole, -NH) $\mathrm{cm}^{-1} ;{ }^{1} \mathrm{H}$ NMR $\left(400 \mathrm{MHz}, \mathrm{DMSO}-d_{6}\right)$ : $\delta 3.89\left(\mathrm{~s}, 3 \mathrm{H}, \mathrm{OCH}_{3}\right), 5.46\left(\mathrm{br}, 2 \mathrm{H},-\mathrm{NH}_{2}\right), 7.21-$ $7.36\left(\mathrm{~m}, 2 \mathrm{H}, \mathrm{Ar}-\mathrm{H}\right.$ of $\left.\mathrm{C}_{7} \& \mathrm{C}_{6}-\mathrm{H}\right), 7.63\left(\mathrm{~d}, 1 \mathrm{H}, \mathrm{C}_{5}-\mathrm{H}\right.$ of Ar-H), $7.79\left(\mathrm{~s}, 1 \mathrm{H}, \mathrm{NH}\right.$ Pyrazole), $8.12\left(\mathrm{~s}, 1 \mathrm{H}, \mathrm{C}_{5}{ }^{\prime}-\right.$ $\mathrm{H}$ of thiazole), 8.64 (s, $1 \mathrm{H}, \mathrm{C}_{4}-\mathrm{H}$ of coumarin); ${ }^{13} \mathrm{C}$ NMR $\left(400 \mathrm{MHz}, \mathrm{DMSO}-d_{6}\right.$ ): $\delta 91.3,116.9,121.4$, 124.1, 125.8, 127.8, 128.5, 129.5, 132.9, 134.8, 146.0, 149.6, 153.9, 156.7, 166.5, MS: $\mathrm{m} / z$ 340. Anal. Calcd for $\mathrm{C}_{16} \mathrm{H}_{12} \mathrm{~N}_{4} \mathrm{O}_{3} \mathrm{~S}$ : C, 56.46; H, 3.55; N, 16.46. Found: C, 56.41; H, 3.51; N, 16.42\%. 
3-(2-(5-amino-1H-pyrazol-4-yl)thiazol-4-yl)-6-chloro-2H-chromen-2-one 3c

White solid. Yield 80\%. m.p. $267-269^{\circ} \mathrm{C}$. IR (KBr): $1606(-\mathrm{C}=\mathrm{N}), 1720$ (lactone, $\left.-\mathrm{C}=\mathrm{O}\right)$ and 3421 (pyrazole, -NH) cm ${ }^{-1} ;{ }^{1} \mathrm{H}$ NMR (400 MHz, DMSO- $\left.d_{6}\right): \delta 5.51\left(\mathrm{br}, 2 \mathrm{H},-\mathrm{NH}_{2}\right), 7.36-7.56(\mathrm{~m}, 2 \mathrm{H}, \mathrm{Ar}-\mathrm{H}$ of $\left.\mathrm{C}_{7} \& \mathrm{C}_{8}-\mathrm{H}\right), 7.79\left(\mathrm{~d}, 1 \mathrm{H}, \mathrm{C}_{5}-\mathrm{H}\right.$ of $\left.\mathrm{Ar}-\mathrm{H}, J=9 \mathrm{~Hz}\right), 7.81\left(\mathrm{~s}, 1 \mathrm{H}, \mathrm{NH}\right.$ Pyrazole), $8.21\left(\mathrm{~s}, 1 \mathrm{H}, \mathrm{C}_{5}{ }^{\prime}-\mathrm{H}\right.$ of thiazole), $8.61\left(\mathrm{~s}, 1 \mathrm{H}, \mathrm{C}_{4}-\mathrm{H}\right.$ of coumarin).

\section{3-(2-(5-amino-1H-pyrazol-4-yl)thiazol-4-yl)-6,8-dichloro-2H-chromen-2-one 3d}

White solid. Yield 81\%. m.p. $260-262^{\circ} \mathrm{C}$. IR (KBr): $1605(-\mathrm{C}=\mathrm{N}), 1723$ (lactone, $\left.-\mathrm{C}=\mathrm{O}\right)$ and 3410 (pyrazole, $-\mathrm{NH}) \mathrm{cm}^{-1} ;{ }^{1} \mathrm{H}$ NMR $\left(400 \mathrm{MHz}, \mathrm{DMSO}-d_{6}\right)$ : $\delta 5.41\left(\mathrm{br}, 2 \mathrm{H},-\mathrm{NH}_{2}\right), 7.61\left(\mathrm{~d}, 1 \mathrm{H}, \mathrm{J}=1 \mathrm{~Hz}, \mathrm{C}_{7} \mathrm{H}\right.$ of Coumarin), $7.88\left(\mathrm{~d}, 1 \mathrm{H}, \mathrm{C}_{5}-\mathrm{H}\right.$ of Ar-H, $\left.J=9 \mathrm{~Hz}\right), 8.10\left(\mathrm{~s}, 1 \mathrm{H}, \mathrm{NH}\right.$ Pyrazole), $8.34\left(\mathrm{~s}, 1 \mathrm{H}, \mathrm{C}_{5}{ }^{\prime}-\mathrm{H}\right.$ of thiazole), $8.66\left(\mathrm{~s}, 1 \mathrm{H}, \mathrm{C}_{4}-\mathrm{H}\right.$ of coumarin).

\section{3-(2-(5-amino-1H-pyrazol-4-yl)thiazol-4-yl)-6-bromo-2H-chromen-2-one 3e}

White solid. Yield 84\%. m.p. $268-270^{\circ}$ C. IR (KBr): $1608(-\mathrm{C}=\mathrm{N}), 1720$ (lactone, $\left.-\mathrm{C}=\mathrm{O}\right)$ and 3414 (pyrazole, $-\mathrm{NH}) \mathrm{cm}^{-1} ;{ }^{1} \mathrm{H}$ NMR (400 MHz, DMSO- $\left.d_{6}\right)$ : $\delta 5.45\left(\mathrm{br}, 2 \mathrm{H},-\mathrm{NH}_{2}\right), 7.31-7.45(\mathrm{~m}, 2 \mathrm{H}, \mathrm{Ar}-\mathrm{H}$ of $\left.\mathrm{C}_{7} \& \mathrm{C}_{8}-\mathrm{H}\right), 7.76\left(\mathrm{~d}, 1 \mathrm{H}, \mathrm{C}_{5}-\mathrm{H}\right.$ of $\left.\mathrm{Ar}-\mathrm{H}, J=9 \mathrm{~Hz}\right), 7.78\left(\mathrm{~s}, 1 \mathrm{H}, \mathrm{NH}\right.$ Pyrazole), $8.46\left(\mathrm{~s}, 1 \mathrm{H}, \mathrm{C}_{5}{ }^{\prime}-\mathrm{H}\right.$ of thiazole), $8.71\left(\mathrm{~s}, 1 \mathrm{H}, \mathrm{C}_{4}-\mathrm{H}\right.$ of coumarin).

\section{3-(2-(5-amino-1H-pyrazol-4-yl)thiazol-4-yl)-6,8-dibromo-2H-chromen-2-one 3f}

White solid. Yield 80\%. m.p. $263-265^{\circ} \mathrm{C}$. IR (KBr): $1610(-\mathrm{C}=\mathrm{N}), 1723$ (lactone, $\left.-\mathrm{C}=\mathrm{O}\right)$ and 3421 (pyrazole, -NH) cm ${ }^{-1} ;{ }^{1} \mathrm{H}$ NMR (400 MHz, DMSO- $\left.d_{6}\right)$ : $\delta 5.43\left(\mathrm{br}, 2 \mathrm{H},-\mathrm{NH}_{2}\right), 7.65\left(\mathrm{~d}, 1 \mathrm{H}, \mathrm{J}=1 \mathrm{~Hz}, \mathrm{C}_{7} \mathrm{H}\right.$ of Coumarin), $7.80\left(\mathrm{~d}, 1 \mathrm{H}, \mathrm{C}_{5}-\mathrm{H}\right.$ of $\left.\mathrm{Ar}-\mathrm{H}, J=9 \mathrm{~Hz}\right), 7.91$ (s,1H, NH Pyrazole), 8.31 (s, 1H, $\mathrm{C}_{5}{ }^{\prime}-\mathrm{H}$ of thiazole), 8.71 (s, 1H, $\mathrm{C}_{4}-\mathrm{H}$ of coumarin).

\section{Synthesis of (E)-3-(dimethylamino)-2-(4-(2-oxo-2H-chromene-3-yl)thiazol-2-yl)but-2-enenitrile(4a- f)}

A mixture of 2-(4-(2-oxo-2H-chromene-3-yl)thiazol-2-yl)acetonitrile 1 a-f (1 mmol), N,N-Dimethyl acetamide dimethyl acetal (DMA-DMA) $(1.5 \mathrm{mmol})$ in toluene was refluxed for 2 hours. Then the reaction mixture was checked by TLC, after completion of reaction the solvent was distilled out and added hexane to get the crude solid, which was filtered, dried and recrystallized from hexane/EtOAc.

(E)-3-(dimethylamino)-2-(4-(2-oxo-2H-chromen-3-yl)thiazol-2-yl)but-2-enenitrile 4a

Brown solid.Yield 88\%. m.p. $258-260^{\circ} \mathrm{C}$. IR $(\mathrm{KBr}): 1608(-\mathrm{C}=\mathrm{N}), 1721$ (lactone, $\left.-\mathrm{C}=\mathrm{O}\right) \quad \mathrm{cm}^{-1} ;{ }^{1} \mathrm{H}$ NMR (400 MHz, DMSO- $\left.d_{6}\right): \delta 2.23\left(\mathrm{~s}, 3 \mathrm{H},-\mathrm{CH}_{3}\right), 3.45\left(\mathrm{~s}, 6 \mathrm{H}, \mathrm{N}\left(\mathrm{CH}_{3}\right)_{2}\right), 7.44-7.60\left(\mathrm{~m}, 2 \mathrm{H}, \mathrm{Ar}-\mathrm{H}\right.$ of $\mathrm{C}_{6} \&$ $\left.\mathrm{C}_{8}-\mathrm{H}\right), 7.66\left(\mathrm{~m}, 1 \mathrm{H}, \mathrm{Ar}-\mathrm{H}\right.$ of $\left.\mathrm{C}_{7}-\mathrm{H}\right), 7.8\left(\mathrm{~d}, 1 \mathrm{H}, \mathrm{C}_{5}-\mathrm{H}\right.$ of $\left.\mathrm{Ar}-\mathrm{H}, J=9 \mathrm{~Hz}\right), 8.46\left(\mathrm{~s}, 1 \mathrm{H}, \mathrm{C}_{5}{ }^{\prime}-\mathrm{H}\right.$ of thiazole), 8.66 (s, $1 \mathrm{H}, \mathrm{C}_{4}-\mathrm{H}$ of coumarin) ${ }^{13} \mathrm{C}$ NMR $\left(400 \mathrm{MHz}\right.$, DMSO- $\left.d_{6}\right): \delta 20.141 .4,78.6,116.4,120.6,124.4$, 125.7, 127.5, 128.5, 129.5, 149.2, 164.2, 166.1, 168.0, MS: $\mathrm{m} / z$ 337. Anal. Calcd for $\mathrm{C}_{18} \mathrm{H}_{15} \mathrm{~N}_{3} \mathrm{O}_{2} \mathrm{~S}: \mathrm{C}$, 64.08; H, 4.48; N, 12.45. Found: C, 64.04; H, 4.45; N, $12.41 \%$.

Synthesis of 3-(2-(5-amino-3-methyl-1H-pyrazole-4-yl)thiazol-4-yl)-2H-chromene-2-one(5a-f)

To a solution of (E)-3-(dimethylamino)-2-(4-(2-oxo-2H-chromene-3-yl)thiazol-2-yl)but-2-enenitrile4a-f $(1 \mathrm{mmol})$, in ethanol was added hydrazine hydrochloride $(1 \mathrm{mmol})$ was refluxed for 8 hours. After that reaction mixture was checked by TLC, then solvent was distilled out under reduced pressure to get the residue to this added methyl teritiarybutylether and filtered insoluble material. This organic layer was washed with $10 \% \mathrm{NaOH}$ solution, followed by dil. $\mathrm{HCl}$ and water. This was dried in sodium sulphate and concentrated to get the crude compound was recrystallized from toluene/EtOAc.

\section{3-(2-(5-amino-3-methyl-1H-pyrazol-4-yl)thiazol-4-yl)-2H-chromen-2-one 5a}

Brown solid. Yield 86\%. m.p. $271-273^{0} \mathrm{C}$. IR $(\mathrm{KBr}): 1610(-\mathrm{C}=\mathrm{N}), 1722$ (lactone, $\left.-\mathrm{C}=\mathrm{O}\right)$ and 3420 (pyrazole, -NH) cm ${ }^{-1} ;{ }^{1} \mathrm{H}$ NMR $\left(400 \mathrm{MHz}, \mathrm{DMSO}-d_{6}\right): \delta 2.34\left(\mathrm{~s}, 3 \mathrm{H},-\mathrm{CH}_{3}\right), 5.46\left(\mathrm{br}, 2 \mathrm{H},-\mathrm{NH}_{2}\right), 7.34-$ $7.46\left(\mathrm{~m}, 2 \mathrm{H}, \mathrm{Ar}-\mathrm{H}\right.$ of $\left.\mathrm{C}_{7} \& \mathrm{C}_{8}-\mathrm{H}\right), 7.60\left(\mathrm{~m}, 1 \mathrm{H}, \mathrm{Ar}-\mathrm{H}\right.$ of $\left.\mathrm{C}_{6}-\mathrm{H}\right), 7.76\left(\mathrm{~d}, 1 \mathrm{H}, \mathrm{C}_{5}-\mathrm{H}\right.$ of Ar-H, $\left.J=9 \mathrm{~Hz}\right), 7.8$ 
(s,1H, NH Pyrazole), 8.36 (s, 1H, $\mathrm{C}_{5}{ }^{\prime}-\mathrm{H}$ of thiazole), 8.71 (s, $1 \mathrm{H}, \mathrm{C}_{4}-\mathrm{H}$ of coumarin); ${ }^{13} \mathrm{C} \mathrm{NMR}(400$ MHz, DMSO- $\left.d_{6}\right): \delta 13.26,94.3,116.4,121.4,124.3,125.8,127.8,128.5,129.3,139.8,146.0,149.5$, 153.9, 156.7, 166.8, MS: $m / z$ 324. Anal. Calcd for $\mathrm{C}_{16} \mathrm{H}_{12} \mathrm{~N}_{4} \mathrm{O}_{2} \mathrm{~S}: \mathrm{C}, 59.25 ; \mathrm{H}, 3.73 ; \mathrm{N}, 17.27$. Found: C, $59.21 ; \mathrm{H}, 3.70 ; \mathrm{N}, 17.24 \%$.

\section{3-(2-(5-amino-3-methyl-1H-pyrazol-4-yl)thiazol-4-yl)-8-methoxy-2H-chromen-2-one5b}

White solid. Yield 80\%. m.p. $273-275^{\circ} \mathrm{C}$. IR (KBr): $1609(-\mathrm{C}=\mathrm{N}), 1720$ (lactone, $\left.-\mathrm{C}=\mathrm{O}\right)$ and 3410 (pyrazole, $-\mathrm{NH}) \mathrm{cm}^{-1} ;{ }^{1} \mathrm{H}$ NMR $\left(400 \mathrm{MHz}, \mathrm{DMSO}-d_{6}\right): \delta 2.36\left(\mathrm{~s}, 3 \mathrm{H}, \mathrm{CH}_{3}\right), 3.86\left(\mathrm{~s}, 3 \mathrm{H}, \mathrm{OCH}_{3}\right), 5.46$ (br,2H, $\left.-\mathrm{NH}_{2}\right), 7.31-7.47\left(\mathrm{~m}, 2 \mathrm{H}, \mathrm{Ar}-\mathrm{H}\right.$ of $\left.\mathrm{C}_{7} \& \mathrm{C}_{6}-\mathrm{H}\right), 7.64\left(\mathrm{~d}, 1 \mathrm{H}, \mathrm{C}_{5}-\mathrm{H}\right.$ of $\left.\mathrm{Ar}-\mathrm{H}\right), 7.74(\mathrm{~s}, 1 \mathrm{H}, \mathrm{NH}$ Pyrazole), 8.10 (s, $1 \mathrm{H}, \mathrm{C}_{5}{ }^{\prime}-\mathrm{H}$ of thiazole), $8.67\left(\mathrm{~s}, 1 \mathrm{H}, \mathrm{C}_{4}-\mathrm{H}\right.$ of coumarin); ${ }^{13} \mathrm{C}$ NMR $\left(400 \mathrm{MHz}, \mathrm{DMSO}-d_{6}\right.$ ): $\delta 13.28,56.2,91.3,113.9,121.4,123.3,124.4,126.9,129.6,139.2,146.2,149.7,153.6,156.7,166.8$, MS: $m / z$ 354. Anal. Calcd for $\mathrm{C}_{17} \mathrm{H}_{14} \mathrm{~N}_{4} \mathrm{O}_{3} \mathrm{~S}: \mathrm{C}, 57.62 ; \mathrm{H}, 3.98 ; \mathrm{N}, 15.81$. Found: $\mathrm{C}, 57.58 ; \mathrm{H}, 3.94 ; \mathrm{N}$, $15.78 \%$.

\section{RESULTS AND DISCUSSION}

In the present protocol, the title compounds have been prepared unlike the literature methods; we synthesized the title compounds $\mathbf{3} \boldsymbol{\&} \mathbf{5}$ in two steps without using cyclization reagent. This reaction takes place here to expand the scope of synthetic transformation and offer a convenient method for 3-(2-(5amino-1H-pyrazole-4-yl)thiazol-4-yl)-2H-chromene-2-one and its derivatives. The title compounds $(\mathbf{3 , 5})$ have been synthesized from (Step-1) readily available starting materials like 2-(4-(2-oxo-2H-chromene-3yl)thiazol-2-yl)acetonitrile (1a-f), with DMF-DMA/DMA-DMA in toluene resulted as uncyclized compounds 2, 4 which on further reaction with hydrazine hydrochloride in ethanol given the title compounds of Scheme-1 and 2.

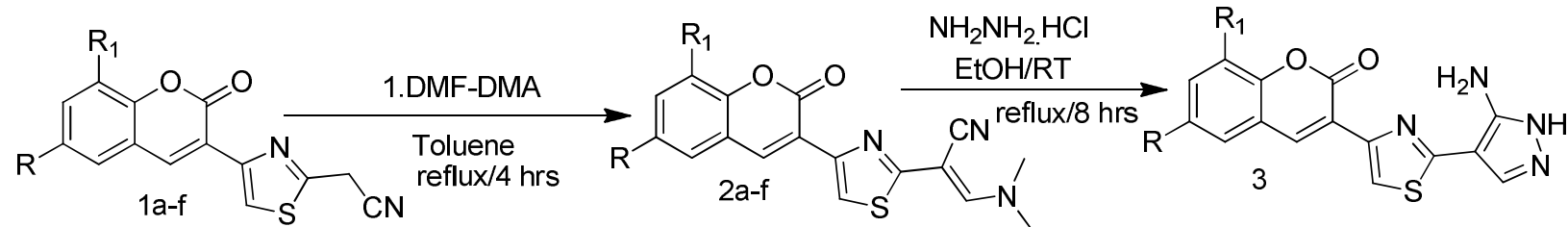
1a: $\mathrm{R}=\mathrm{R}_{1}=\mathrm{H} \quad \mathrm{d}: \mathrm{R}=\mathrm{R}_{1}=\mathrm{Cl}$
b: $\mathrm{R}=\mathrm{H}, \mathrm{R}_{1}=\mathrm{OCH}_{3} \mathrm{e}: \mathrm{R}=\mathrm{Br}, \mathrm{R}_{1}=\mathrm{H}$
c: $R=C l, R_{1}=H \quad$ f: $R=R_{1}=B r$
2a: $R=R_{1}=H$
$\mathrm{d}: \mathrm{R}=\mathrm{R}_{1}=\mathrm{Cl}$
$\mathrm{d}: \mathrm{R}=\mathrm{R}_{1}=\mathrm{Cl}$
b: $R=H, R_{1}=O_{3}$ e: $R=B r, R_{1}=H \quad$ b: $R=H, R_{1}=O C H_{3}$ e: $R=B r, R_{1}=H$
c: $\mathrm{R}=\mathrm{Cl}, \mathrm{R}_{1}=\mathrm{H} \quad$ f: $\mathrm{R}=\mathrm{R}_{1}=\mathrm{Br}$
c: $\mathrm{R}=\mathrm{Cl}, \mathrm{R}_{1}=\mathrm{H} \quad \mathrm{f}: \mathrm{R}=\mathrm{R}_{1}=\mathrm{Br}$
3a: $R=R_{1}=H$

Scheme-1

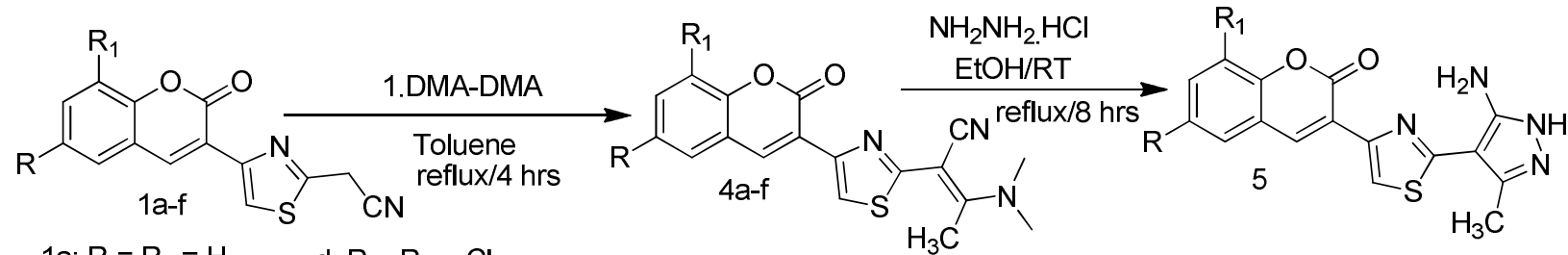

4a: $\mathrm{R}=\mathrm{R}_{1}=\mathrm{H} \quad \mathrm{d}: \mathrm{R}=\mathrm{R}_{1}=\mathrm{Cl} \quad$ 5a: $\mathrm{R}=\mathrm{R}_{1}=\mathrm{H}$ b: $R=H, R_{1}=O_{3} H_{3}$ e: $R=B r, R_{1}=H$ b: $R=H, R_{1}=O C H_{3}$ e: $R=B r, R_{1}=H$ C: $R=C l, R_{1}=H \quad$ f: $R=R_{1}=B r \quad$ c: $R=C l, R_{1}=H \quad$ f: $R=R_{1}=B r$

Scheme-2

The yields of products (Scheme-1 and 2) are 80-90\%. The entire synthesized materials were characterized by analytical and spectral data for various representative compounds $\mathbf{2 a}, \mathbf{4 a}$, and $\mathbf{3 a - f}, \mathbf{5 a}-\mathbf{b}$ have given. The IR spectra of compound 2a confirmed prominent peaks at $1608(-\mathrm{C}=\mathrm{N}), 1720$ (lactone, $\mathrm{C}=\mathrm{O}$ ), $\mathrm{cm}^{-1}$, in the ${ }^{1} \mathrm{HNMR}$ spectra six protons appears as a singlet around $\delta 2.84$ for two methyl groups, $\mathrm{C}=\mathrm{CH}$ proton is appeared as a singlet at $\delta 6.86$. The mass spectrum of $2 \mathbf{a}$ confirmed the molecular ion at $\mathrm{m} / \mathrm{z}$ 323. The IR spectra of compound 3a confirmed prominent peaks $1607(-\mathrm{C}=\mathrm{N}), 1719$ (lactone, $-\mathrm{C}=\mathrm{O}$ ), 3418 (pyrazole, $-\mathrm{NH}$ ) $\mathrm{cm}^{-1}$, consistent with the assigned structures. The ${ }^{1} \mathrm{H}$ NMR (DMSO- $d_{6}$ ) spectrum of 
3a confirmed signals around $\delta 5.41\left(\mathrm{~b}, 2 \mathrm{H}, \mathrm{NH}_{2}\right), 7.34-7.46(\mathrm{~m}, 2 \mathrm{H}, \mathrm{Ar}-\mathrm{H}), 7.61\left(\mathrm{~m}, 1 \mathrm{H}\right.$, of $\mathrm{C}_{6}-\mathrm{H}$ coumarin), $7.74\left(\mathrm{~d}, 1 \mathrm{H}, \mathrm{Ar}-\mathrm{H}\right.$ of $\left.\mathrm{C}_{5}-\mathrm{H}\right), 7.79\left(\mathrm{~s}, 1 \mathrm{H}, \mathrm{NH}\right.$ of pyrazole), $8.1\left(\mathrm{~s}, 1 \mathrm{H}, \mathrm{C}_{5}\right.$ '- $\mathrm{H}$ of thiazole) and $8.68\left(\mathrm{~s}, 1 \mathrm{H}, \mathrm{C}_{4}-\mathrm{H}\right.$ coumarin), in the mass spectrum 3a confirmed the molecular ion peak at $\mathrm{m} / \mathrm{z} 310$ $(100 \%)$.

The IR spectra of compounds 4a confirmed prominent peaks at 1606 $(-\mathrm{C}=\mathrm{N}), 1721$ (lactone, $-\mathrm{C}=\mathrm{O}$ ), $\mathrm{cm}^{-1}$, in the ${ }^{1} \mathrm{HNMR}$ spectra $=\mathrm{CCH}_{3}$ protons appear as a singlet at $\delta$ 2.86, the remaining six protons appeared at $\delta 3.46$ as a singlet. The mass spectrum of 4 a confirmed the molecular ion at $\mathrm{m} / \mathrm{z} 337$. The IR spectra of compounds 5a showed prominent peaks $1610(-\mathrm{C}=\mathrm{N}), 1722$ (lactone, $-\mathrm{C}=\mathrm{O}$ ), 3420 (pyrazole, $-\mathrm{NH}$ ) $\mathrm{cm}^{-1}$, consistent with the assigned structures. The ${ }^{1} \mathrm{H}$ NMR $\left(\mathrm{DMSO}-d_{6}\right)$ spectrum of $\mathbf{5 a}$ confirmed signals around $\delta 2.34\left(\mathrm{~s}, 3 \mathrm{H},-\mathrm{CH}_{3}\right), 5.46\left(\mathrm{~b}, 2 \mathrm{H}, \mathrm{NH}_{2}\right), 7.34-$ 7.46(m, 2H, Ar- $\mathrm{H}), 7.60\left(\mathrm{~m}, 1 \mathrm{H}\right.$, of $\mathrm{C}_{6}-\mathrm{H}$ coumarin), $7.76\left(\mathrm{~d}, 1 \mathrm{H}, \mathrm{Ar}-\mathrm{H}\right.$ of $\left.\mathrm{C}_{5}-\mathrm{H}\right), 7.8(\mathrm{~s}, 1 \mathrm{H}, \mathrm{NH}$ of pyrazole), $8.36\left(\mathrm{~s}, 1 \mathrm{H}, \mathrm{C}_{5}\right.$ '- $\mathrm{H}$ of thiazole) and $8.68\left(\mathrm{~s}, 1 \mathrm{H}, \mathrm{C}_{4}-\mathrm{H}\right.$ coumarin), in the mass spectrum $5 \mathbf{a}$ confirmed the molecular ion peak at $\mathrm{m} / \mathrm{z} 324(100 \%)$.

\section{Biological Activity}

\section{Antibacterial Activity}

The entire prepared compounds (Table-1) 3a-f and 5a-f were screened for antibacterial activity by using agar well diffusion method ${ }^{24}$ against bacterial species (Gram-positive and Gram-negative) were cultured on nutrient agar plates at $37^{\circ} \mathrm{C}$. A plate containing $20 \mathrm{ml}$ of nutrient agar was spread with $100 \mu \mathrm{l}$ of culture. Ciprofloxacin has been used as a standard drug for the evaluation of antibacterial activity. Compounds in table-1, 3a, b, $\mathbf{c}$ and 5a, b, $\mathbf{c}$ were found to be active for all bacterial strains. While the substitution of chloro groups at the $6^{\text {th }}$ position of coumarin gives satisfactory results.In the case of Grampositive bacteria methoxy group at $8^{\text {th }}$ position, coumarin increases the bacterial activity of the compounds. In the case of Gram-negative bacteria substitution of a methoxy grouping at the $8^{\text {th }}$ position of coumarin decreases the antibacterial activity of compounds.

Table-1: Antibacterial Activity Data of Compounds 3a-f and 5a-f

\begin{tabular}{|c|c|c|c|c|c|}
\hline \multirow{2}{*}{ Compound } & \multirow{2}{*}{$\begin{array}{l}\text { Conc. } \\
(\mu \mathrm{g} / \mathrm{ml})\end{array}$} & \multicolumn{4}{|c|}{ Antibacterial Activity Diameter of Zone of Inhibition (in $\mathrm{mm}$ ) } \\
\hline & & S.aureus & B.subtilis & E. coli & S.paratyphib \\
\hline $3 \mathrm{a}$ & 100 & 17 & 18 & 16 & 15 \\
\hline $\mathrm{b}$ & 100 & 19 & 20 & 17 & 16 \\
\hline $\mathrm{c}$ & 100 & 18 & 19 & 20 & 19 \\
\hline $\mathrm{d}$ & 100 & 15 & 18 & 14 & 16 \\
\hline $\mathrm{e}$ & 100 & 11 & 10 & 12 & 13 \\
\hline $\mathrm{f}$ & 100 & 16 & 14 & 15 & 16 \\
\hline $5 a$ & 100 & 17 & 18 & 17 & 18 \\
\hline$b$ & 100 & 20 & 20 & 16 & 15 \\
\hline $\mathrm{c}$ & 100 & 17 & 19 & 18 & 19 \\
\hline $\mathrm{d}$ & 100 & 10 & 14 & 13 & 16 \\
\hline $\mathrm{e}$ & 100 & 13 & 16 & 12 & 11 \\
\hline $\mathrm{f}$ & 100 & 12 & 15 & 17 & 14 \\
\hline $\begin{array}{l}\text { Ciprofloxacin } \\
\text { (Stand.) }\end{array}$ & 100 & 20 & 20 & 25 & 25 \\
\hline
\end{tabular}

\section{CONCLUSION}

This protocol mostly describes the antibacterial activity for title compounds (3a-f and 5a-f) and its derivatives. Some of these synthesized compounds (3a, b, c and 5a, b, c) have been confirmed a wide range of potentially promising antibacterial activity. The remarkable lead of this procedure are good yields $(80-90 \%)$, little reaction times, fair reaction conditions, easy workup procedure, and finally simple preparation and using without a catalyst for the cyclization.

\section{ACKNOWLEDGMENT}

The authors are thankful to the Director NIT Warangal for analytical, spectral data and special thanks to IICT Hyderabad for the Mass spectral data support. 


\section{REFERENCES}

1. G. Turan-Zitouni, P. Chevallet, F.S. Kilic, K. Erol, Eur. J. Med. Chem, 35(6), 635 (2000), DOI: 10.1016/S0223-5234(00)00152-5

2. (a) T.A. Farghaly, M.A. Abdallah, M.A. Khedr et al., J. Heterocyclic. Chem., 54(4), 2417 (2017), DOI:10.1002/jhet.2838; (b) A. Tabbi,Z. A. Kaplancikli, D. Tebbaniet al., Turk. J. Chem., 40, 641 (2016)

3. (a) E. L. Luzina, A. V. Popov, Eur. J. Med. Chem., 44(12), 4944(2009), DOI: 10.1016/j.ejmech.2009.08.007; (b)T. I. de Santana,M. de. O. Barbosa, P.A.T. de M Gomes etal.,Eur. J. Med. Chem.,144, 874 (2018), DOI: 10.1016/j.ejmech.2017.12.040

4. N. Singh, B. Sudhir Kumar, AshokKumar, Eur. J. Med. Chem, 43(11), 2597 (2008), DOI: 10.1016/j.ejmech.2007.12.024

5. R. K. Rawal, R. Tripathi, S. B. Katti et al., Eur. J. Med. Chem., 43(12), 2800(2008), DOI:10.1016/j.ejmech.2007.12.015

6. T. Iino, D. Tsukahara, K.Kamata, et al., Bioorg. Med. Chem,17(7), 2733 (2009), DOI:10.1016/j.bmc.2009.02.038

7. A. Satoh,Y. Nagatomi,Y. Hirataet al., Bioorg. Med. Chem. Lett.,19(18), 5464 (2009), DOI:10.1016/j.bmcl.2009.07.097

8. S. Micheal, G. S. Sattari,F. Jamali, J. Pharm. Pharmaceut. Sci.,4(1), (2001)

9. J. V. Greenhill, In Comprehensive Heterocyclic Chemistry, 5, 305 (1984)

10. X. Zhi,G. Chuan,R. Qing-Cheng,S. Xu-Feng, F. Lian-Shun, Lv. Zao-Sheng, Eur. J. Med. Chem.,139, 429 (2017), DOI:10.1016/j.ejmech.2017.07.059

11. M. F. Ahmed, A. K. AliKorany, M.A. Taha, EI-Debss, S.M. Abdelrahman, E.A. Abdel-Galil,A. Naglaa, Abdel- Hafez, M. Mohamed Abdulla, Eur. J. Med. Chem., 45, 5887 (2010), DOI:10.1016/j.ejmech.2010.09.054

12. I. H. Ahmed, S.A. Ahmed, A. Youssef Kamal, S.I. Kandeel Wael,Abou-Elmagd, Eur. J. Med. Chem,42, 934 (2007), DOI:10.1016/j.ejmech.2006.12.032

13. (a) Ch. Simpal, P. Sarvesh, Ch. Rajani, Synthetic Communications, 44(10), 1333 (2014), DOI:10.1080/00397911.2013.837186; (b) Harish Kumar, S. Deepika, J. Sandeep, J. Neelam, Eur. J. Med. Chem, 70, 248(2013), DOI:10.1016/j.ejmech.2013.10.004

14. V. Rajeswar Rao,P. Vijaya Kumar, Synthetic Communications, 36(15), 2157 (2006), DOI: $10.1080 / 00397910600637012$

15. V. Rajeswar Rao, T. V. Padmanabha Rao, Indian. J. Chem., 25B, 413 (1986)

16. M. J. Matos, S.V. Rodriguez, L. E. Santana, C. F. Uriarte, Y. Edfu, A. Santos, Munoz-Crego, Med. Chem, 8, 1140 (2012), DOI:10.2174/157340612804075205

17. S.Yuan, Z. Cheng-He, Bioorg. Med. Chem. Lett., 21, 956 (2011), DOI:10.1016/j.bmcl.2010.12.059

18. C. Fsahin, M. Sajak and Ertan, FABAB, Farm. Org., 13, 365(1998), Steering I T \& Worthington P A, PCT/N/APP/WO, 93, 08, 1991, 180. Chem. Abstr., 119, 1993, 180765K.

19. Y. Bansal, P. Sethi, G. Bansal, Med. Chem. Res., 22, 3049 ( 2013)

20. B. Mahantesha, B. J. Vishwanath, N. B. Nivedita, S. L. Sandeep, D. Venkatesh,D.Narayanachar, Eur. J. Med. Chem., 74, 225 (2014), DOI:10.1016/j.ejmech.2013.12.061

21. T. Anuradha, S. Ramit, J. Vikas, Eur. J. Med. Chem, 101, 476 (2015), DOI:10.1016/j.ejmech.2015.07.010

22. H. Yuan-Qiang, X. Zhi, Z. Shu,W. Xiang, D. Jun-Wei,L. Zao-Sheng, F. Lian-Shun, Eur. J. Med. Chem, 136, 122 (2017), DOI:10.1016/j.ejmech.2017.05.004

23. B. Alessandra, C. Concettina, R. Angela, F. Giovanna, S. Azzurra, B. Federica, M.C. Rita, D. Martino, M. Emil, M. Alessia, L. Stefano, G. Silvia, European Journal of Medicinal Chemistry, 127, 577(2017), DOI:10.1016/j.ejmech.2017.01.020

24. S. K. Sharma, A.P. Singh, Der. Pharmacia. Lett., 3, 427 (2011)

[RJC-5375/2019] 